\title{
Effect of Anisotropy of Cellulose Nanocrystal Suspensions on Stratification, Domain Structure Formation, and Structural Colors
}

Konrad W. Klockars, ${ }^{\dagger}$ Blaise L. Tardy, ${ }^{*}{ }^{\dagger}$ Maryam Borghei, ${ }^{\dagger}$ Anurodh Tripathi, ${ }^{\dagger}{ }^{\S \odot}$ Luiz G. Greca, ${ }^{\dagger}$ and Orlando J. Rojas $*,+, \pm, \S \subset$

\author{
${ }^{\dagger}$ Department of Bioproducts and Biosystems, School of Chemical Engineering, and ${ }^{\ddagger}$ Department of Applied Physics, School of \\ Science, Aalto University, FI-00076 Aalto, Finland \\ ${ }^{\S}$ Department of Chemical and Biomolecular Engineering, North Carolina State University, Raleigh, North Carolina 27695, United \\ States
}

Supporting Information

ABSTRACT: Outstanding optical and mechanical properties can be obtained from hierarchical assemblies of nanoparticles. Herein, the formation of helically ordered, chiral nematic films obtained from aqueous suspensions of cellulose nanocrystals (CNCs) were studied as a function of the initial suspension state. Specifically, nanoparticle organization and the structural colors displayed by the resultant dry films were investigated as a function of the anisotropic volume fraction (AVF), which depended on the initial CNC concentration and equilibration time. The development of structural color and the extent of
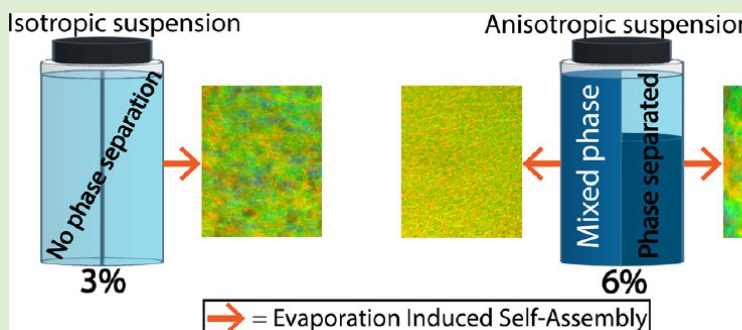

$=$ Evaporation Induced Self-Assembly macroscopic stratification were studied by optical and scanning electron microscopy as well as UV-vis spectroscopy. Overall, suspensions above the critical threshold required for formation of liquid crystals resulted in CNC films assembled with longer ranged order, more homogeneous pitches along the cross sections, and narrower specific absorption bands. This effect was more pronounced for the suspensions that were closer to equilibrium prior to drying. Thus, we show that high AVF and more extensive phase separation in CNC suspensions resulted in large, long-range ordered chiral nematic domains in dried films. Additionally, the average $\mathrm{CNC}$ aspect ratio and size distribution in the two separated phases were measured and correlated to the formation of structured domains in the dried assemblies.

\section{INTRODUCTION}

Cellulose nanocrystals (CNCs) offer a significant opportunity to form hierarchically structured materials mimicking the most robust materials observed in nature. ${ }^{1,2} \mathrm{CNCs}$ are generally obtained by hydrolysis of plant or marine biomass, ${ }^{3}$ resulting in highly crystalline and rodlike assemblies with dimensions ranging from 3 to $70 \mathrm{~nm}$ in width and 35 to $3000 \mathrm{~nm}$ in length. ${ }^{3}$ CNCs are biodegradable and low to nontoxic, ${ }^{4}$ and their size and surface charge depend on the source and isolation protocol (hydrolysis severity, redispersion, purification steps, etc.). ${ }^{5-7}$ Besides their outstanding chemical resistance, CNCs also display thermal stability, making them ideal building blocks for the formation of next-generation materials. ${ }^{8-10}$

Mechanically, individual CNCs are extremely robust, with an elastic modulus of the order of $10^{2} \mathrm{GPa} .{ }^{11,12}$ The assembly of $\mathrm{CNCs}$ into hierarchically structured materials enables the mechanical properties of individual $\mathrm{CNCs}$ to translate into tough materials with added functional properties, such as specific light reflections, fracture dissipation, and control over mesoporous properties. ${ }^{13,14}$ Because of their inherent aspect ratio and chiral nature, $\mathrm{CNC}$ suspensions self-assemble into chiral nematic $(\mathrm{CN})$ liquid crystals above a critical concentration, which depends on the CNC size and charge as well as the properties of the liquid medium $(\mathrm{pH}$, temperature, ionic strength, electrolyte type, composition, among others). ${ }^{5,6,15}$ Upon drying by evaporation-induced self-assembly (EISA), the chiral nematic order observed in the suspension may be preserved, ${ }^{7,16,17}$ resulting in films that display structural colors, which are produced through the selective reflection of light. The observation of structural colors is directly correlated to the long-range order of CNCs required for mechanically robust superstructures. Therefore, the optical properties of CNC films provide insight into their architecture and mechanical properties.

Understanding the transition from liquid crystals to solid films is paramount to control the development of long-ranged chiral nematic order, ${ }^{16}$ where CNCs are aligned within each of the planes and helically arranged across them. ${ }^{1,18-21}$ Numerous studies exist on the formation of the chiral nematic liquid crystalline phase in CNC suspensions through EISA, but the systematic evaluation of the transition from liquid crystals to dry films has only been scarcely addressed. ${ }^{2-25}$ The same factors affecting the critical concentration also influence the pitch of the chiral nematic order in CNC suspensions. ${ }^{6,15,16,26}$

Received: March 21, 2018

Revised: $\quad$ May 11, 2018

Published: May 12, 2018 
Moreover, the chiral nematic order and pitch observed in solid CNC films are closely correlated to those observed in liquid crystals, although they are significantly shorter in the dry state and, regularly, have a larger long-range order. The transition from liquid crystals to solids with helical order also depends on other factors, such as drying shear, ${ }^{24,27}$ topography, ${ }^{28}$ or surface chemistry of the substrate used to support the formation of the film. ${ }^{29}$ Further understanding of the correlation between the liquid crystalline and dry states is required to produce CNC materials homogeneously organized across large areas, such as CNC films with a constant pitch size or with a controlled pitch gradient throughout the film.

Herein, we systematically evaluated the effect of the anisotropic volume fraction (AVF) of liquid crystalline CNC suspensions on the optical properties and long-range order of dry CNC films formed via EISA. Additionally, we evaluated $\mathrm{CNC}$ segregation by size and size polydispersity as a function of the state of equilibration of the liquid crystalline CNC suspension, i.e., the role of AVF on the long-range order and optical properties of CNC films. For this evaluation, our experiments included CNC films produced from suspensions with identical total solid content but varied initial concentrations. The film morphology, pitch size, and optical properties were characterized by scanning electron microscopy (SEM), optical microscopy, and UV-vis spectroscopy.

In contrast to CNC films formed from suspensions at initial concentrations below the critical concentration, we found that those formed from suspensions above the critical threshold required for the formation of liquid crystals $(\text { AVF }>0)^{22}$ displayed longer ranged order. This effect was even more pronounced when suspensions with concentrations above the critical concentration were left to equilibrate prior to EISA. In addition, the resultant films exhibited a more distinct gradient of pitches along the cross section, resulting in narrower specific absorption bands, as evidenced by UV-vis. These effects were significantly less pronounced for films formed from suspensions below the critical concentration. The structural domains and extent of macroscopic (micrometer-scale) stratification in CNC films formed by EISA were thoroughly evaluated by SEM as a function of initial AVF.

We also demonstrate that the size and polydispersity of suspended CNCs affected the formation of domains and pitches in a single $\mathrm{CNC}$ film. We show this relation by evaluating the size distribution of CNCs (transmission electron microscopy) in the anisotropic and isotropic phases of an equilibrated liquid crystalline suspension. The work presented herein shines light on the transitions that occur from liquid crystals to hierarchically structured materials, which is necessary for the formation of large area, uniform CNC materials with controlled long-range order in the macroscale. The results also bear meaning to the formation of structured materials from liquid crystalline suspensions formed from other mesogens.

\section{EXPERIMENTAL SECTION}

2.1. Materials. The cellulose nanocrystals, CNCs (CAS No. 778920-0), were isolated via sulfuric acid hydrolysis from dissolving-grade wood fibers and obtained as a concentrated slurry after dilution and membrane filtration (typical dimensions of $5-20 \mathrm{~nm}$ in width and $150-200 \mathrm{~nm}$ in length and sulfur content of $0.95 \mathrm{wt} \%=297 \mathrm{~mm} / \mathrm{kg}$ ). They were produced as an aqueous suspension $(11.1 \% \mathrm{w} / \mathrm{w})$ at the USDA's Forest Products Laboratory (FPL, Madison, WI) and acquired through the Process Development Center in the University of Maine. Millipore (Synergy UV) Milli-Q water was used to dilute the CNC suspension. Ethanol (A, 94\%) was obtained from Altia Oyj.
2.2. Evaluation of Anisotropic Volume Fraction Development. The $11.1 \%(\mathrm{w} / \mathrm{w}) \mathrm{CNC}$ suspension was used as received and diluted with Milli-Q water to concentrations of $1,2,3,4,5$, and $6 \%$ $(\mathrm{w} / \mathrm{w})$ and stored at $6{ }^{\circ} \mathrm{C}$ until use. Diluted suspensions ( $55 \mathrm{~mL}$ each) were poured into tall, sealable vials (diameter $2.6 \mathrm{~cm}$, height $12.5 \mathrm{~cm}$ ) to investigate the evolution of the AVF over time. The glass vials were examined after 7,35 , and 287 days at rest $\left(22-23{ }^{\circ} \mathrm{C}\right)$. The position of the boundary between the isotropic and anisotropic phase was monitored and subsequently normalized with respect to the total suspension height, yielding the AVF. In additional experiments, a $6 \%$ CNC suspension was placed into a syringe $(60 \mathrm{~mL})$, in a vertical position, and kept undisturbed for 28 days. The self-assembled anisotropic and isotropic phases were subsequently separated and the solids content $(\mathrm{w} / \mathrm{w})$ of each sample was measured.

2.3. Film Formation via EISA. Petri dishes (polystyrene, diameter $=3.5 \mathrm{~cm}$ ) were exposed to ozone for $30 \mathrm{~min}$, using a Biofore Nanosciences UV-ozone chamber, after which they were thoroughly washed and rinsed with ion-exchanged water for $30 \mathrm{~min} .{ }^{30}$ Another set of Petri dishes were treated for films reported in the Supporting Information (Figure S1), the only difference being an ethanol wash instead of a water wash. A KSV Instruments CAM 200 optical contact angle meter was used to measure the static contact angle of a $7 \mu \mathrm{L}$ water droplet on the Petri dishes before and after ozone treatment and rinsing.

For film casting, fixed volumes of CNC aqueous suspension (stored for 28 days) were added to the treated Petri dishes with a diameter of $3.5 \mathrm{~cm}(4,3,2.4$, or $2 \mathrm{~mL}$ of the $3,4,5$, or $6 \% \mathrm{CNC}$ suspension, respectively). An identical set of suspensions were cast in Petri dishes, using CNC suspensions that were isolated and "equilibrated" by sealing the Petri dishes for 7 days prior to EISA. Films from the two sets of samples (not equilibrated and equilibrated) were then formed by drying $\left(21^{\circ} \mathrm{C}\right.$ and $20 \%$ humidity) for $2-4$ days, depending on the $\mathrm{CNC}$ concentration. For simplicity, the films obtained after equilibration ("E") for 7 days and subsequently dried are denoted as E3, E4, E5, and E6, where the numeral refers to the initial suspension concentration $(3,4,5$, or $6 \%$ CNCs). The respective set of films that were dried immediately upon casting (nonequilibrated samples, "N") are denoted as N3, N4, N5, and N6.

Finally, another set of films were prepared by casting separately anisotropic and isotropic phases. These were extracted from an aqueous suspension of CNCs (6\% initial concentration) that was held undisturbed for 28 days to induce phase separation. For this set, approximately $2 \mathrm{~mL}$ of each of the two separated phases was cast in the respective Petri dish and further equilibrated for 7 days prior to drying. The volume used for each suspension, the isotropic, "iso", and anisotropic, "an", phases, was adjusted to account for the small differences in CNC concentration in each of the suspensions. Following the nomenclature used, the obtained films are labeled as $E_{a n} 6$ and $E_{i s s} 6$. As a control, another set of samples were dried immediately from the separated fractions, without any equilibration. These samples are thereafter referred to as $\mathrm{N}_{a n} 6$ and $N_{i s o} 6$. A summary of the nomenclature followed is provided in Table 1.

2.4. CNC Film and Substrate Characterization. The separated anisotropic and isotropic phases of the $\mathrm{CNC}$ suspensions were each diluted to obtain a CNC concentration of 0.001 wt \%. A 300-mesh carbon-coated, ultrathin copper grid was first plasma-treated for $20 \mathrm{~s}$ to make the carbon surface hydrophilic. The grid was then dipped into the $0.001 \mathrm{wt} \%$ suspension for $5 \mathrm{~s}$ and then dried in ambient conditions on a filter paper for a few minutes. The prepared sample was imaged in a $120 \mathrm{kV}$ FEI Tecnai 12 transmission electron microscope. The distribution of CNC lengths was obtained by sizing 100 particles in each sample using ImageJ software. A histogram was generated with a bin size of $20 \mathrm{~nm}$ for the isotropic fraction and a bin size of $50 \mathrm{~nm}$ for the anisotropic fraction. The sizes of the CNCs measured by TEM (Figure $6 \mathrm{~b}-\mathrm{c}_{2}$ ) were within the range of those measured previously for the same nanocrystals obtained from prefractionated suspensions. ${ }^{31-34}$ Figure S2 shows additional TEM images of the two fractions used for the size distribution calculations and an example of the parameters used for obtaining the Gaussian fit. 
Table 1. Nomenclature Used To Describe the CNC Cast Films and Corresponding Casting Conditions

\begin{tabular}{lccc} 
sample & $\begin{array}{c}\text { equilibration } \\
\text { (days) }\end{array}$ & $\begin{array}{c}\text { casting suspension } \\
\text { concn }(\%)\end{array}$ & CNC suspension phase \\
$\mathrm{N} 3$ & 0 & 3 & $\begin{array}{c}\text { preseparation "pristine" } \\
\text { suspension }\end{array}$ \\
$\mathrm{E} 3$ & 7 & 4 & \\
$\mathrm{~N} 4$ & 0 & 5 & \\
$\mathrm{E} 4$ & 7 & 6 & \\
$\mathrm{~N} 5$ & 0 & & \\
$\mathrm{E} 5$ & 7 & & \\
$\mathrm{~N} 6$ & 0 & & separated isotropic phase \\
$\mathrm{E} 6$ & 7 & & separated anisotropic \\
$\mathrm{N}_{i s o} 6$ & 0 & & \\
$E_{i s o} 6$ & 7 & & \\
$\mathrm{~N}_{a n} 6$ & 0 & & \\
$E_{a n} 6$ & 7 & & \\
\hline
\end{tabular}

The $\mathrm{pH}$ was measured with a Mettler Toledo SevenEasy $\mathrm{pH}$ meter from a $5.5 \%$ suspension at $25{ }^{\circ} \mathrm{C}$, and a value of 6.6 was obtained. The zeta potential $(-32.7 \mathrm{mV})$ was obtained using a Malvern nano Series Zetasizer by diluting the $\mathrm{CNC}$ suspension to a final concentration of $0.25 \% \mathrm{CNC}$ and $10 \mathrm{mM}$ sodium chloride. An apparent CNC size of $68.5 \mathrm{~nm}$ was obtained by dynamic light scattering (Malvern Nano Series Zetasizer) using a $0.025 \%$ suspension. The correlogram obtained indicated a well-dispersed suspension as suggested by the unimodal profile and a sharp transition between high and low correlation coefficients (Figure S3). ${ }^{35}$ The results are presented in Discussion S1 together with a summary and discussion of the properties of the used commercial, wood-derived CNCs.

Photographs of films were taken outdoors with a digital camera at a resolution of 4640 by 2610 pixels against a black background, from the base of the films (facing upward) through the transparent Petri dish substrate. Transmission spectra were obtained with a PerkinElmer Lambda $950 \mathrm{UV}$-vis spectrometer. Three positions in the central part of each film were measured, all at an equal distance from the center. The averaged transmission data are used in the results.

$\mathrm{CNC}$ films were fractured and their cross sections at the center region (away from edges) were imaged with a Sigma Zeiss ULTRAplus scanning electron microscope (SEM). Prior to imaging, the samples were sputtered to create a $4 \mathrm{~nm}$ platinum coating on the fractured surfaces. Microscope images were taken at 10 times magnification using a Leica DM4500 P microscope in reflection mode.

2.5. Pitch Measurement. The pitch was obtained as an average of six measurement points from the bottom section of each of the SEM images of the films (E3, E6, and N6, Figure $4 a-c_{3}$ ). The values obtained should be taken as approximations given the challenges of locating areas with strictly equal nanocrystal directors and because the $\mathrm{CN}$ pseudoplanes might not be perfectly parallel with the viewing axis. ${ }^{36}$ It has previously been suggested that measurement bias associated with tilted areas can be minimized if one reports the lowest pitch value. However, this technique was employed only in Figure $4 a_{3}$, as we cannot rule out that the pitch size measured in different areas of Figure $4 b_{3}-c_{3}$ in fact resulted from actual pitch size differences due to stratification or domains, as reported by Dumanli et al. ${ }^{23}$ Therefore, while for Figure $4 a_{3}$ the pitch values were obtained only from the upper section, with lower brightness, in the case of Figures $4 b_{3}-c_{3}$ they were measured from the whole image.

\section{RESULTS AND DISCUSSION}

3.1. Evolution of the Anisotropic Volume Fraction (AVF). Figure 1 shows a schematic illustration of the evolution of the anisotropic volume fraction (AVF). The relative height (volume) was measured by identifying the boundary between the two phases. For the 1, 2, and $3 \%$ suspensions, $A V F=0$, which is why only the $3 \%$ suspension is displayed in Figure 1.
Phase separation occurred for CNC suspensions prepared at a concentration higher than 3\%. Furthermore, Figure S4 shows that the AVF is reduced over time for each suspension. The 4 and $5 \%$ suspensions phase separated after 1 week, while the $6 \%$ suspensions required a longer time. This behavior is due to the inherent apparent viscosity of the suspensions as a function of concentration and the larger volume of liquid crystals at higher concentrations. ${ }^{15}$ The relatively longer times needed for phase separation compared to other studies can be attributed to the larger CNC suspension volumes used in our study. ${ }^{6,15,37,38}$

3.2. CNC Films. The development of chiral nematic $(\mathrm{CN})$ ordering during the transition from $\mathrm{CNC}$ suspension to dry films was thoroughly investigated as a function of initial CNC suspension concentration and equilibration. The films were dried from suspensions of 3, 4, 5, and 6\%, as per Figure 1. One batch of samples was equilibrated for 7 days prior to drying, while a second batch was dried immediately after casting. In the experiments, the CNC total mass was kept the same and therefore the films had the same areal mass $(120 \mathrm{mg}$ in 9.6 $\mathrm{cm}^{2}$ ). Bearing in mind that there may exist an uneven distribution of film thickness in the cast area, any reported difference in film thickness for the same position on the plane of the film (e.g., central part of the film) is ascribed to variations in the packing density within the films. As expected, the drying time decreased with increasing initial concentration. The ozone treatment carried out on the Petri dishes significantly increased the surface energy (water contact angle of $42.4^{\circ}$ compared to $87.6^{\circ}$ for untreated dishes) and ensured homogeneous adhesion with the forming CNC film. Previous studies have reported on the effects of initial CNC concentration on the macroscopic radial color evolution ${ }^{39}$ and equilibration time on the color shift of dry films. ${ }^{25}$ This work provides a systematic evaluation of the effect of the initial CNC suspension AVF (Figure 1 and Figure S4) on the color and the cross-sectional stratification in dried films.

Figure 2 shows microscope images of the films, taken in reflection mode, in which the color arises from the selective reflection of left-handed circularly polarized light, according to eq $1:{ }^{40}$

$$
\lambda_{\mathrm{r}}=n_{\mathrm{av}} \frac{p}{2} \sin \theta
$$

where $n_{\mathrm{av}}$ is the refractive index, $p$ is the pitch, and $\theta$ is the angle of incidence. The reflection is caused by chiral nematic (CN) domains that are assumed to have a given pitch size. ${ }^{23,40}$ The longer evaporation time needed for the more diluted CNC suspension (3\% CNC concentration) resulted in a film with larger and more uniform $\mathrm{CN}$ domains (Figure 2a), whereas the shorter evaporation time for the more concentrated, $6 \% \mathrm{CNC}$ suspension produced a film with smaller domains (Figure 2d). Indeed, upon evaporation, the sample assembled from the 6\% CNC suspension was expected to reach more rapidly kinetic arrest or gelation ${ }^{16,41}$ and did not have time to rearrange into a longer range order, i.e., larger domains. These observations for samples that were not equilibrated are reminiscent of previous results. ${ }^{24}$ Equilibration was expected to be essential in $\mathrm{CN}$ formation, especially for samples with large AVF, which take a longer time for phase separation, as shown in Figure S4. Indeed, the samples that underwent equilibration after casting and before drying displayed larger domain sizes as a function of initial concentration (Figure $2 \mathrm{e}-\mathrm{h}$ ). We should note the effect of mixing caused by pipetting upon casting. Subsequently, after 
Cellulose nanocrystal concentration (w/w)
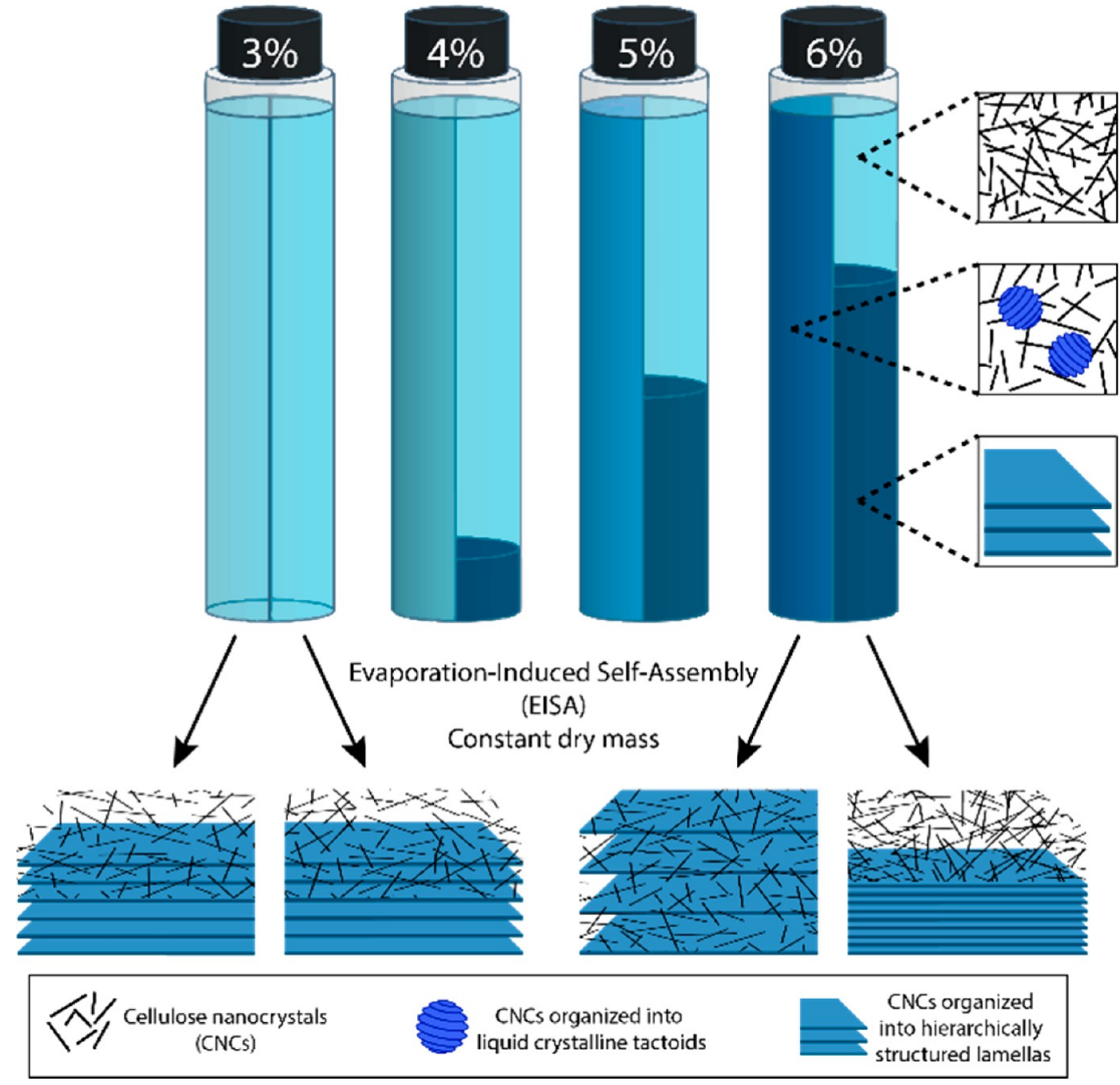

Figure 1. Schematic design of the experimental setup. The left side of the vials correspond to CNC aqueous suspensions soon after mixing at the given concentration. The right side of the vials correspond to cellulose nanocrystal suspensions after 35 days of equilibration. Phase separation occurs into anisotropic (dark blue, bottom phase) and isotropic (light blue, upper phase) phases. The AVF corresponds to the volume of the bottom anisotropic phase, where the CNCs are organized as liquid crystals, normalized to the total volume of the suspension. Note: as for the $3 \% \mathrm{CNC}$ suspension, those at lower concentrations than $3 \%$ also yield $\mathrm{AVF}=0$. The different $\mathrm{CNC}$ suspensions as a whole (mixed and after equilibration) were cast and dried in Petri dishes. Dry supported films obtained from CNC suspensions with initial concentrations of 3 and $6 \%$ are displayed schematically (bottom of the figure), showing the ordering in the out-of-plane direction. Note that the spacing between the blue "sheets" in the schematics of Figure 1 (and Figure 5) is used only for illustration purposes since no such discontinuity exists in reality. These sheets are meant to correspond to chiral nematic pseudoplanes, where each $\mathrm{CNC}$ is oriented in the same direction.

rest inside of the Petri dish, the mixed suspension may have relaxed into a more equilibrated state.

$\mathrm{UV}$-vis spectroscopy provides information about the average pitch size of the ordered regions within the film. Figure S5 shows transmittance spectra, measured from the eight films. However, the meaningfulness of the data is perturbed for samples N6 and E6 by topographical inhomogeneity in the films (Figure 3d,f). Those stresses are a product of the adhesion properties between CNCs as well as between the CNCs and the substrate that are developing during the final stages of drying. Herein, we observe that higher residual stress may develop during EISA during EISA from highly ordered suspensions when compared to less ordered suspensions. Therefore, UV-vis analysis is most meaningful for samples N/ E3-5. The samples obtained from equilibrated initial CNC concentrations of 3,4 , and $5 \%$ had a higher nonspecific \% transmission (measured at $700 \mathrm{~nm}$ ) than the nonequilibrated ones: $70 \%(\mathrm{~N} 3) \rightarrow 77 \%(\mathrm{E} 3) ; 70 \%(\mathrm{~N} 4) \rightarrow 77 \%(\mathrm{E} 4) ; 63 \%$ $(\mathrm{N} 5) \rightarrow 74 \%$ (E5). Note that the difference is more pronounced for the high initial AVF samples. Furthermore, the equilibrated films (E3, E4, E5) were consistently blueshifted, in line with work by Tran et al., ${ }^{25}$ except for the E6 film. Notably, compared to the N6 film, E6 had a narrower transmittance band and steeper derivative of the transmittance in the same band, indicating a narrower distribution of pitch size. In contrast, there was no difference in the derivatives of the spectral peak of the N3 and E3 films (Figure $\mathrm{SSc}_{2}$ ), indicating a similar distribution of pitch sizes.

Figure 3 shows photographs of all the dried films, which have a radial evolution of the structural color. Generally, drying sessile drops tend to form films that red-shift radially toward the film edges ${ }^{24,27,42}$ due to faster drying rates toward the edges 

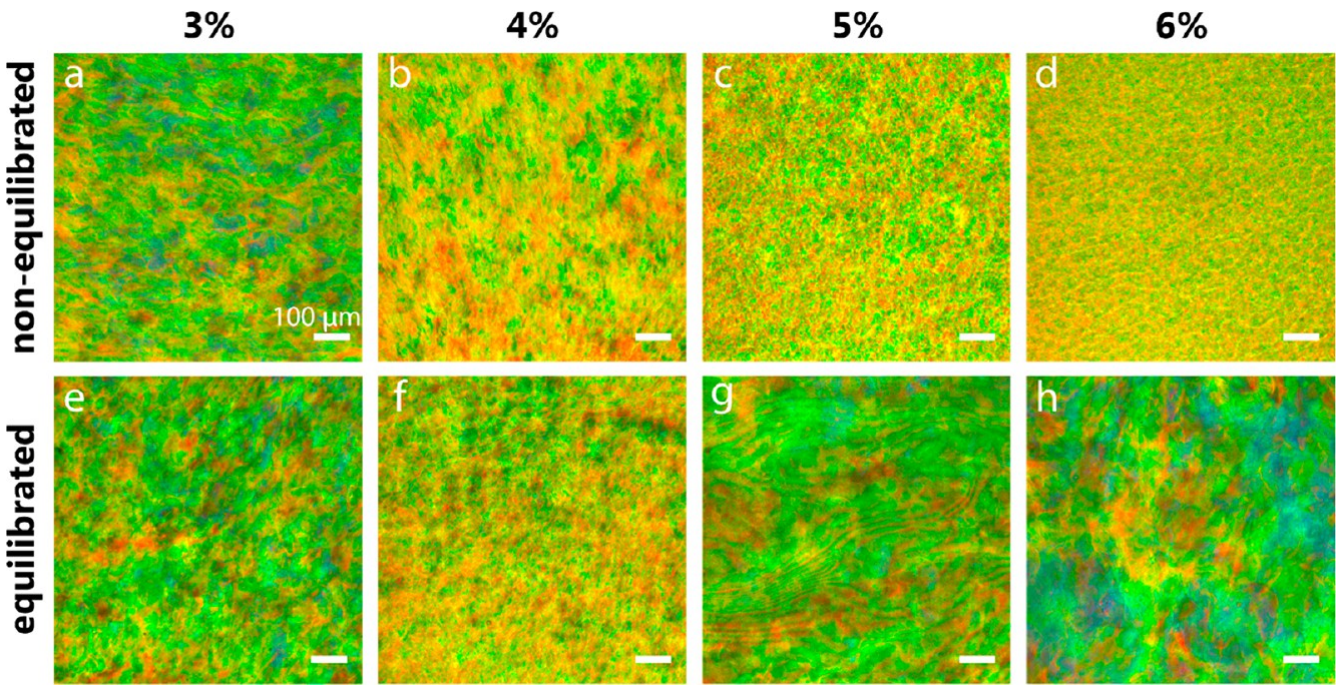

Figure 2. Reflected-light microscopy images obtained with the light beam incident perpendicular to the film plane. (a-d) CNC films obtained directly after drying for 2 (d) to 4 days (a). (e, f) CNC films obtained after equilibrating the suspension for 7 days postcasting and prior to drying. The microscope images correspond to samples (a) N3, (b) N4, (c) N5, (d) N6, (e) E3, (f) E4, (g) E5, and (h) E6. The scale bars correspond to 100 $\mu \mathrm{m}$.
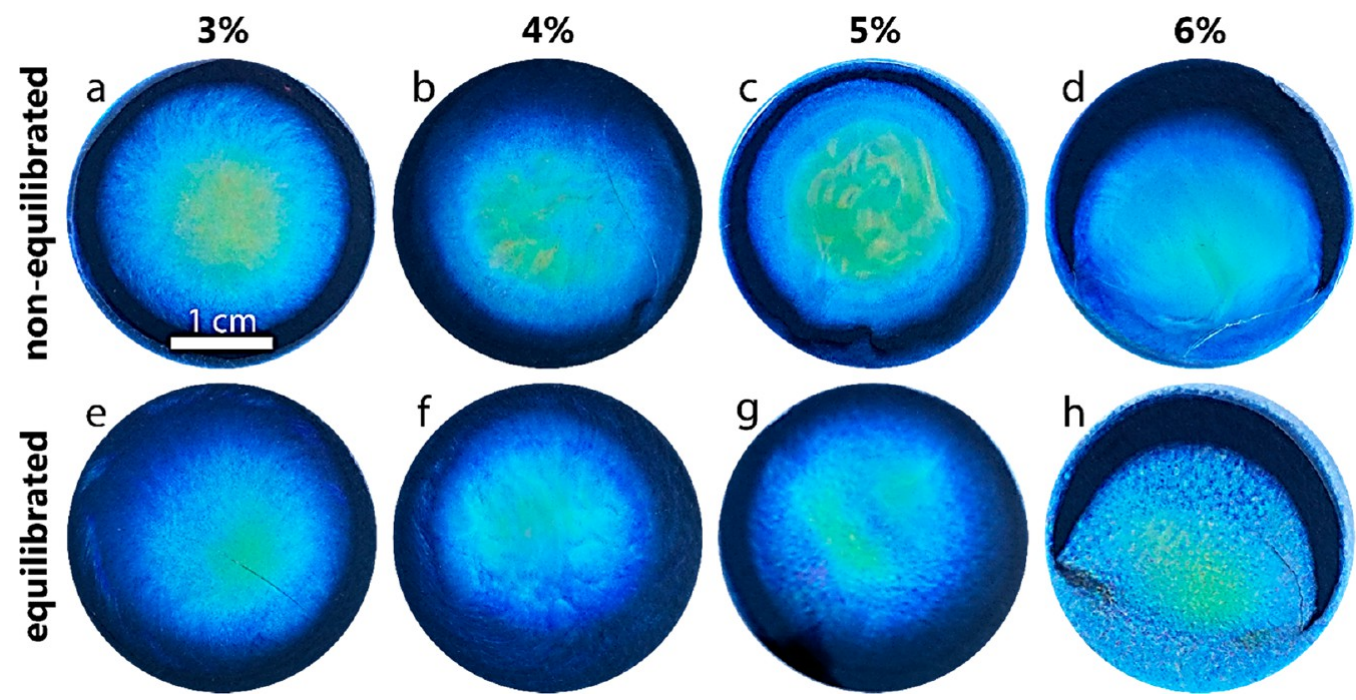

Figure 3. Photographs of CNC dry films (a) N3, (b) N4, (c) N5, (d) N6, (e) E3, (f) E4, (g) E5, and (h) E6 films, taken with a black background.

(responsible for the coffee ring effect). ${ }^{42}$ However, our films blue-shifted toward the edges due to effects of the walls of the Petri dish, which has been observed earlier. ${ }^{24,25}$ The central yellow-green area was smaller in all of the equilibrated films, except for the E6 sample. This observation is consistent with the blue-shift in UV-vis transmission spectra of the equilibrated films (Figure S5).

Comparisons of microscopy and photographic images of CNC films (Figures 2 and 3) reveal that as the initial CNC concentration increases, equilibration becomes increasingly important to obtain large $\mathrm{CN}$ domains. In contrast, equilibration is not as a consequential factor in films produced in the absence of the anisotropic phase. This observation is further supported by observation of the films shown in Figure S1, which formed on substrates with a similar wettability (contact angle $=40.0^{\circ}$ ) but a different surface chemistry and equilibration time ( 3 days). Therefore, we propose that equilibration mainly acts on the AVF, which produces a domain architecture that depends on the initial state of the suspension, prior to the EISA. At concentrations slightly above the critical concentration, $\mathrm{CN}$ formation is limited to small tactoids, $^{22}$ which coalesce into larger color-generating $\mathrm{CN}$ domains as the concentration increases, e.g., upon EISA. Thus, the long-range transitions are time- and concentration-dependent. Films assembled from suspensions of low CNC concentration $^{43-47}$ undergo a reduction in the specific reflection of structural colors because the evaporating suspensions existed too briefly in the regime between the critical and gelation concentrations. In other words, the drying time limits the extent of domain formation in suspensions that did not contain tactoids prior to the EISA. These effects have been addressed by lowering the evaporation rate. ${ }^{25,43,47}$ However, the nanoparticle mobility promoted by the drying shear in the suspension may still hinder the formation of domains $^{39,48}$ to a greater extent than in an equilibrating CNC suspension. In previous studies, films have been assembled from high AVF suspensions, ${ }^{24,49}$ which should contain tactoids or domains that are responsible for the color generation. 


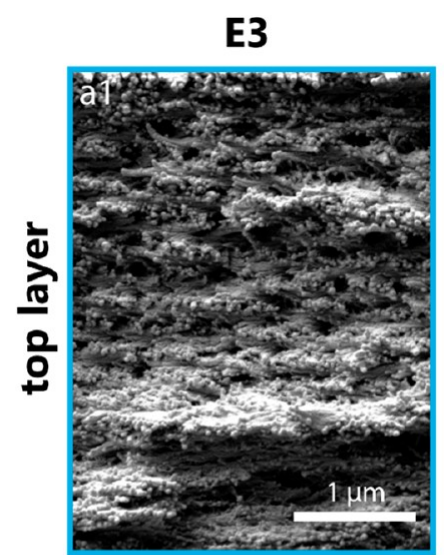

$\Delta$
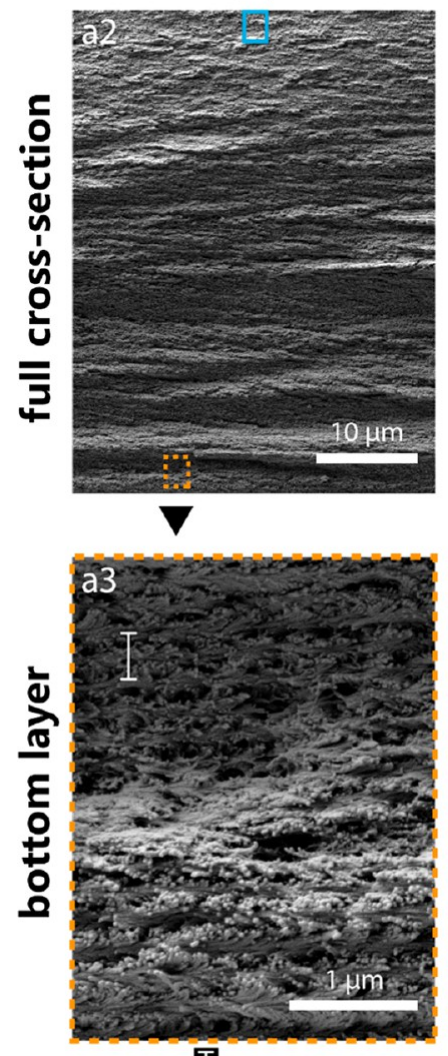

$395 \mathrm{~nm}$
N6

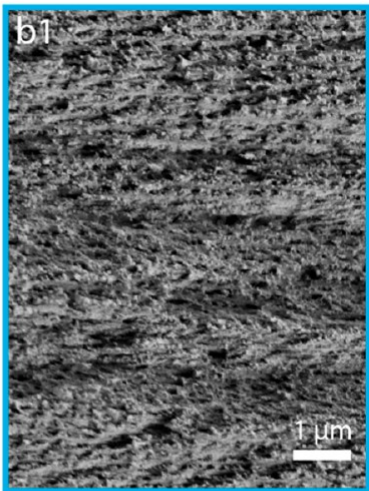

$\Delta$
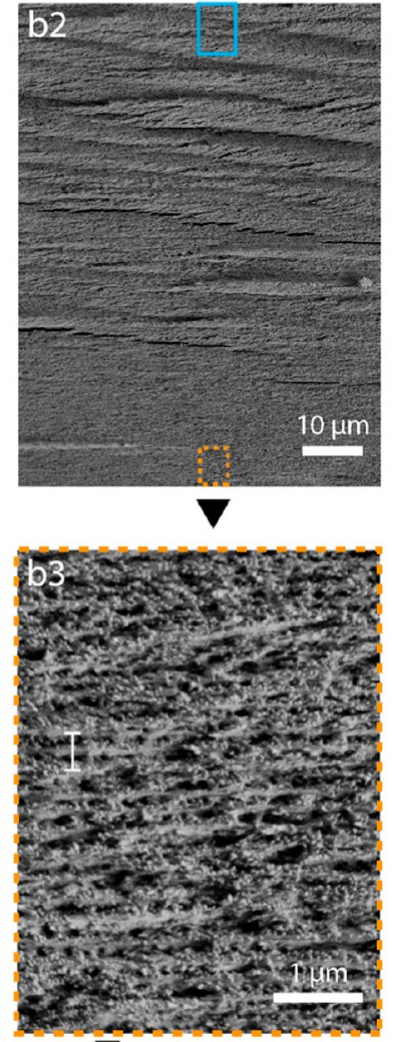

$440 \pm 140 \mathrm{~nm}$
E6

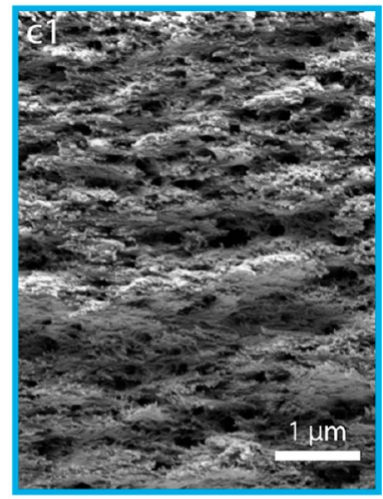

$\boldsymbol{\Delta}$

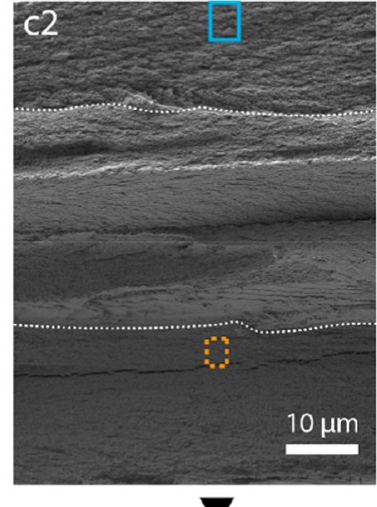

$\nabla$

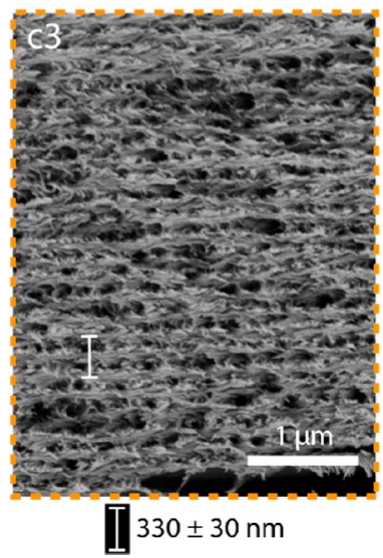

Figure 4. SEM images of the cross sections taken at the center of films fractured. The samples correspond to films E3 ( $\left.a_{1-3}\right)$, E6 ( $\left.b_{1-3}\right)$, and N6 $\left(c_{1-3}\right)$. The full cross section of the films is shown in $\left(a_{2}-c_{2}\right)$ while the top-most sections (facing the air interface, indicated with the blue/continuous line boxes) are displayed in $\left(a_{1}-c_{1}\right)$ at higher magnifications. Likewise, the bottom-most part of the cross section (close to the supporting solid), shown with the orange/dashed line boxes, are shown in $\left(a_{3}-c_{3}\right)$.

However, when transferring the CNC suspension to the solvent casting substrate, for example, by using a pipet, the resultant inherent mixing disrupts the $\mathrm{CN}$ order. Indeed, Gray and $\mathrm{Mu}^{49}$ found that weak shear forces in concentrated (9.6\%) CNC suspension untwists the helical $\mathrm{CN}$ order. On the other hand, the same authors observed nematic-like textures under the observation of a microscope with the sample held between cover glasses. A relaxation transition was subsequently observed from the initially nematic to chiral nematic nanocrystals, over a period of $18 \mathrm{~h}$. Similar effects are at play during suspension transfer into Petri dishes, particularly for high AVF, which display a significantly high viscosity. ${ }^{48-50}$ In our case, the E6 film was obtained from a $6 \%$ suspension that relaxed back to the equilibrated chiral nematic state after 7 days (Figure 1 and Figure S2) prior to drying. As a result, E6 displayed colorful reflections that can be appreciated in Figure $2 \mathrm{~h}$. All our films were cast in a low humidity $(20 \% \mathrm{RH})$, leading to relatively fast drying rates. ${ }^{51}$ The fast drying rate limited the contribution of relaxation during the pregelling stage of the film casting.

3.3. Film Structure and Stratification. SEM images obtained for the films shown in Figure 2d,e,h provided further insights into the distribution of the $\mathrm{CN}$ pitch across the films as a function of the processing protocol (Figure 4). The cross sections taken at the center of the fractured film were imaged, considering that this central area displays the highest ordering. ${ }^{13,23}$ The second row of Figure 4 (Figure $4 a_{2}-c_{2}$ ) 
shows the full cross section of the E3, N6, and E6 films, whereas the upper (Figure $4 a_{1}-c_{1}$ ) and lower (Figure $4 a_{3}-c_{3}$ ) rows correspond to images acquired at high magnification in the top-most and bottom-most zones across the thickness of the films. The images for the E3 film (Figure $4 a_{1-3}$ ) are in good agreement with structural observation made for films assembled from low concentration CNC suspensions. ${ }^{43-47}$ From Figure 2, the limited effect of equilibration on the E3 sample can be clearly appreciated. In comparison, the long-range order across the films N6 (Figure $4 \mathrm{~b}_{1-3}$ ) and E6 (Figure $4 c_{1-3}$ ) clearly emphasizes the effect of equilibration.

Considering the full cross-section view in the middle row of Figure 4, there is a clear difference in the stratification behavior between the samples. This refers to the presence of segments or strata, which vary as one goes from the bottom to the top of the film in the out-of-plane direction. In Figure $4 c_{2}$, there are three distinct strata, separated by the dotted lines. This behavior is in stark contrast to film E3 (Figure $4 a_{2}$ ) and N6 (Figure $4 b_{2}$ ), which showed no significant evidence of stratification in the same direction. Moreover, the cross sections of the films obtained at the highest concentration ( $6 \%$ CNCs), nonequilibrated (N6) and equilibrated (E6), displayed cleaner or sharper fracture surfaces compared to that of film E3. Note, however, that this observation is not as evident considering the middle segment of the E6 cross section. We hypothesize that the presence of large, potentially fusing, tactoids resulted in large domains that caused such discontinuity in the fractured cross section. The large anisotropic volume fraction in the $6 \%$ suspensions, prior to the EISA, led to a more homogeneous helical structure in these films (E6 and N6), effectively making the fracture surface smoother.

The distinct CN structure is evident in all the highmagnification SEM images taken at the bottom-most section of the films (Figure $4 a_{3}-c_{3}$ ). The pitch is shown with vertical lines within the bottom section of the films (see section 2.5 for details). The pitch gives qualitative evidence that the E6 film was the most ordered, with the lowest pitch $(\sim 330 \mathrm{~nm})$, while the N6 film had the highest pitch $(\sim 440 \mathrm{~nm})$. Surprisingly, using the pitch value from Figure $4 c_{3}$, the calculated eq 1 reflection corresponds to the ultraviolet $(258 \mathrm{~nm})$ range. ${ }^{52}$ This characteristic could provide an opportunity to make UV reflectors out of homogeneous CNC films with low pitch values throughout the film. Pitches have been measured from SEM images in previous studies, giving values in the ranges of 300$380 \mathrm{~nm}^{23}$ and $316-354 \mathrm{~nm},{ }^{53}$ which are comparable to the values obtained from film E6. In the present study, the pitch values in dry films appear to increase with higher AVF for nonequilibrated suspensions (N6 vs E3). This is in contrast to the trend that is anticipated for pitch measurements in the precursor suspensions, as a function of AVF. ${ }^{15,16,38}$ Nevertheless, the pitch decreases when comparing the dry films made from equilibrated suspensions (E6 vs E3), following the trend of previous work. This is a better comparison, since in both cases, i.e. in the suspension or in the dry state, equilibrated conditions were considered.

Finally, considering the films obtained from a higher CNC suspension concentration ( $6 \% \mathrm{CNCs}$ ), EISA gave rise to lower pitch values $(330 \mathrm{~nm})$ if the sample was equilibrated (E6), whereas it produced higher pitch values $(440 \mathrm{~nm})$ for nonequilibrated suspensions (N6). The high pitch for the N6 film compared to both E6 and E3 was likely caused by the shorter time window between casting and gelation for the N6 sample (a few hours for N6, versus several hours for E3, and several days for E6 due to equilibration). However, these results need to be taken with caution given the very large scatter in pitch figures, as high as $140 \mathrm{~nm}$ for N6.

While the E3, E6, and N6 films showed increased CN order in their bottom-most section, there were differences for the topmost sections, facing the air interface (Figure $4 a_{1}-c_{1}$ ). Generally, the top parts of $\mathrm{CNC}$ films are more disordered than the bottom parts. ${ }^{27,54,55}$ Faster drying at the surface of the liquid volume causes gelation and setting of the dry films at an earlier stage. ${ }^{16,41,48}$ The top layer solidifies into a more disordered state, ${ }^{27,39,56}$ whereas the bottom part, toward the solid support, is more ordered; here, the effective or local drying rate is slower, and the $\mathrm{CN}$ axis tends to align vertically (in the out-of-plane direction). ${ }^{22,24}$ This vertical gradient of order is apparent for the E3 film (Figure $4 a_{1-3}$ ), in agreement with previous reports. ${ }^{24,27}$ Figure $4 \mathrm{a}_{1}$ shows a highly deformed and disordered helical structure, whereas the bottom layer (Figure $4 \mathrm{a}_{3}$ ) has a more homogeneous $\mathrm{CN}$ order. This difference between the layers is even more pronounced in the E6 film.

A rationalization to the above observations stems from the fact that tactoids tend to form in later stages of the EISA in the isotropic volume fraction, thereby preventing the formation of long-range order in the top part of the film during the short time period prior to kinetic arrest of the suspension (see Figure $4 c_{1}$ for E6). In addition, the E6 film has a clear segmented and discontinuous structure (Figure $4 c_{1,3}$ ), with a more irregular structure in the top, in contrast to the more homogeneous and regular arrangement in the bottom. Figure S6 includes a SEM image of a protruding domain from the middle layer of the E6 film (Figure $4 c_{2}$ ), where the chiral nematic order is apparent. Most interestingly, these domains cause the discontinuous fracture of film E6. The clearly segregated structure in E6 is in drastic contrast with the continuous change evident in film E3, from ordered to disordered structures (Figure $4 a_{1-3}$ ). The ratio of the thickness of the disordered and ordered layers of E6 (Figure $4 c_{2}$ ) roughly corresponds with the ratio of the isotropic versus the anisotropic volume fraction in suspension (Figure 1). This relationship can be explained assuming that the ordered region in the bottom of E6, Figure $4 c_{3}$, forms principally from the anisotropic phase, while the disordered region, Figure $4 c_{1}$, forms from the isotropic phase. Additionally, the isotropic phase of the E6 suspension may contribute to both the upper, disordered region of the film and partially to the middleordered region, where $\mathrm{CNCs}$ form tactoids at an intermediate stage during EISA. In contrast, the N6 film had an almost entirely ordered structure across its thickness but with a larger pitch and pitch size distribution (Figure $4 b_{3}$ ), likely resulting from a mixture of the isotropic and anisotropic phases.

3.4. Domain Structure. Herein, we provide further insights into differences in the long-range order in the E6 and N6 films. The schematics in Figure 5 includes a visualization of the formation of $\mathrm{CN}$ domains and the layered structure of the films as a function of the initial state of the CNCs (whether close to or far from equilibrium) before the EISA. Wang et al. ${ }^{22}$ have explained elegantly how tactoids form and grow from a suspension with an initial $4.1 \%$ CNC concentration, eventually coalescing/fusing to produce large $\mathrm{CN}$ domains, which subsequently flatten into oblate spheroids or ellipsoids. A SEM image of the E6 film reveals $\mathrm{CN}$ domains resulting from partially merged tactoids, as indicated by the yellow dotted lines in Figure $5 a_{1}$, without apparent alignment in the long-range ordered $\mathrm{CN}$ matrix surrounding them. Figure $5 \mathrm{a}_{5}$ further 

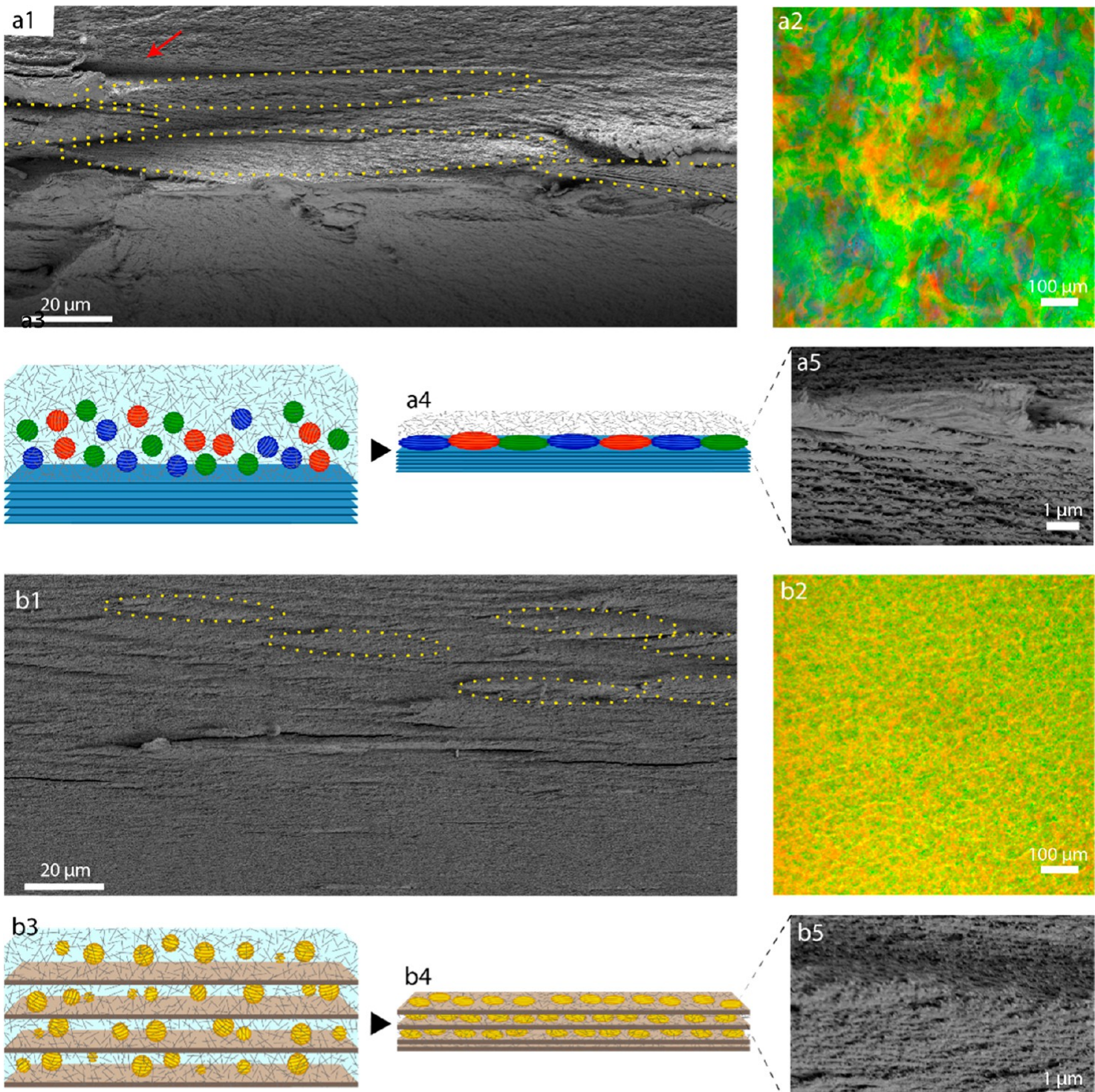

b4
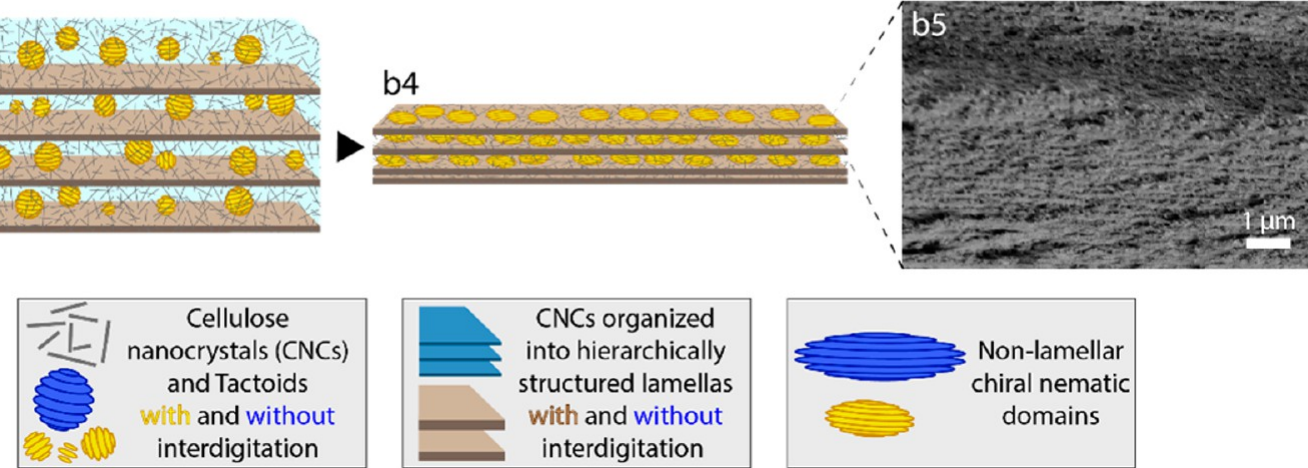

Figure 5. Illustration of chiral nematic domains from film cross sections: $\left(a_{1-5}\right)$ corresponds to the E6 film while $\left(b_{1-5}\right)$ corresponds to N6. $\left(a_{1}\right)$ and $\left(b_{1}\right)$ are low-magnification SEM images of the cross section of E6 and N6, respectively while the corresponding plane-view optical microscope images are included in $\left(a_{2}\right)$ and $\left(b_{2}\right)$ (see Figure 2). The yellow dotted ovals in $\left(a_{1}\right)$ and $\left(b_{1}\right)$ are used to highlight regions comprising large CN domains. The red arrows indicate fusion defects and the blue arrow an area from where a domain was detached upon fracture. $\left(a_{3-4}\right)$ and $\left(b_{3-4}\right)$ are schematics of proposed EISA from the precursor aqueous suspension $\left(a_{3}, b_{3}\right)$ into dry assembled films $\left(a_{4}, b_{4}\right)$. Therein, the spheres (left) and ellipsoids (right) represent $C N$ domains which are appreciated in $\left(a_{5}, b_{5}\right)$ in high-magnification SEM images.

reveals that the domains are of chiral nematic order. The red arrow in Figure $5 a_{1}$ shows a void in the film where an initially present ellipsoidal domain was removed upon film fracture. Interestingly, the size of the planar elliptical domains agrees with that of the colored regions seen in the corresponding optical image of the E6 film (Figure $5 \mathrm{a}_{2}$ ). Figure S7 further displays the segmented structure of the E6 film, containing large domains in the middle part. Each domain has a specific pitch and orientation, which reflects light with wavelengths according to eq 1 . The variation in the pitch and orientation of each $\mathrm{CN}$ domain explains the observed mosaic or tessellation of colors in Figure $5 a_{2}$. Accurate pitch values are difficult to measure from within the $\mathrm{CN}$ domains due to tilting, as observed more clearly in Figure $5 \mathrm{a}_{5}$. The tilt would also result in deviations of the actual viewing angle $\left(<90^{\circ}\right)$, effectively blue-shifting the reflection wavelength of the created color. This observation highlights that the orientation of the domains is significantly affected by the presence of phase boundaries in the liquid crystal suspension prior to EISA.

A hypothetical schematic illustration to explain the evolution of chiral nematic structures during drying is offered in Figure $5 a_{3-4}$, for the central part of the film, where the meniscus is 
assumed to be macroscopically horizontal. Figure $5 \mathrm{a}_{3}$ illustrates the equilibrated condition of the suspension after 7 days. The units drawn in blue correspond to the "lamellar" helical order within the anisotropic volume fraction of the phase-separated CNC suspension. Herein, lamellar corresponds to the longrange ordered, continuous phase within which each sheet represents a $180^{\circ}$ rotation of the chiral nematic director, as depicted in the schematics shown in Figure 5. The colors of the ellipsoidal chiral nematic domains represent the colors seen in the microscope images (Figure $5 \mathrm{a}_{2}, \mathrm{~b}_{2}$ ). Wang et al. ${ }^{22}$ observed that most of the AVF forms a continuous lamellar phase in suspension, where the chiral nematic axis is perpendicular to the plane of the substrate. The spheres in the schematics represent liquid crystalline tactoids in the isotropic phase, with randomly oriented chiral nematic axes. When this suspension starts to dry, following the route Figure $5 a_{3} \rightarrow 5 a_{4}$, the tactoids merge into domains near the isotropic/anisotropic phase boundary and align with the continuous lamellar phase, which shrinks as the $\mathrm{CN}$ planes approach each other and the pitch decreases. ${ }^{51}$ Tactoids observed in suspensions ${ }^{57}$ and captured in resins from suspensions ${ }^{22}$ were spherical. However, the $\mathrm{CN}$ domains observed in Figure $5 \mathrm{a}_{1}$ are distinctly ellipsoidal. These domains were likely flattened by the effect of packing or anisotropic shrinkage ${ }^{22}$ during the assembly. This is supported by the considerably larger pitches observed in the wet state for CNC suspensions compared to those for dry CNC films. ${ }^{16}$ Figure $5 \mathrm{a}_{5}$ further highlights the elliptical or disc-shaped $\mathrm{CN}$ domain of the E6 film. Wang et al. ${ }^{22}$ observed domains solidified from a suspension with $\mathrm{CN}$ axes parallel with both the short and long axes of the ellipsoids. In our assembled films, the $\mathrm{CN}$ axis is parallel only with the short axis of the domain. It remains to be verified whether the domains assume their elliptical shape before or after their $\mathrm{CN}$ axes align with that of the lamellar phase, although anisotropic shrinkage and flattening explains the sphere-to-ellipsoid transformation if the axes align first. The subtle tilt of the elliptical domains in Figure $5 a_{1}$ likely stem from the horizontal pitch difference of underlying domains. The domains sink in areas with initially higher pitch values, which shrink during assembly to a larger extent compared to those with an initially low pitch. The less ordered part of the isotropic volume fraction formed a thick layer of disordered structures on the top of the film, represented by the gray $\mathrm{CNCs}$ in Figure $5 \mathrm{a}_{4}$. The resultant film possesses a segmented structure, Figure $4 c_{2}$, with a continuous ordered helical arrangement in the base, large elliptical and ordered domains in the middle section, and a less ordered top section of the film.

The SEM image of the N6 film in Figure $5 b_{1}$ shows a quite different structure. The continuous helical order gives rise to smaller elliptical formations in the top part of the film, as illustrated by the yellow dotted lines. They are clearly smaller, more numerous, and less distinct than the domains in the E6 film (Figure $5 a_{1}$ ). Figure $5 b_{5}$ further includes a SEM image of the chiral nematic order within the smaller elliptical formation. The color structure observed from the microscope image (Figure $5 b_{2}$ ), a mosaic with smaller characteristic color areas, is quite different from that in Figure $5 a_{1}$. Dumanli et al. ${ }^{23}$ previously observed chiral nematic domains in assembled films with horizontal dimensions in the range of $2-20 \mu \mathrm{m}$, sizes which were comparable to those of the elliptical formations observed in N6 film (Figure $5 b_{1}$ ) but far from the corresponding domains in film E6 (Figure $5 a_{1}$ ). The absence of equilibration and the lower concentration of the starting
CNC suspension (4.1\%) may explain the limited size of the domains in the film produced by Dumanli et al. ${ }^{23}$

Figure $5 b_{3-4}$ displays schematically the possible evolution of the system during the EISA for a nonequilibrated $6 \%$ suspension into a solid film. The transfer of the suspension into a Petri dish may induce enough shearing to unwind any $\mathrm{CN}$ liquid crystal arrangement into a more nematic-like order ${ }^{49}$ (Figure $5 b_{3}$ ). Chen and Gray ${ }^{58}$ measured the interfacial tension between the isotropic and anisotropic phases and obtained values ranging from $1.9 \times 10^{-4}$ to $8.3 \times 10^{-4} \mathrm{mN} \mathrm{m}^{-1}$, which is very low. Furthermore, the interfacial tension increased as the concentration increased. Therefore, the isotropic and anisotropic phases might become highly interdigitated even under weak shearing, effectively disrupting the long-range continuity. In our case, the sample starts drying from a nonequilibrated and mixed phase condition, following Figure $5 b_{3} \rightarrow 5 b_{4}$. The nature of any lamellar and/or tactoidal order (mixed or separated) in the wet state as described in Figure $5 b_{3}$ remains uncertain, although the formations are likely highly interdigitated from the mixed anisotropic and isotropic phases. It is expected that the sample enters the kinetically arrested glassy state ${ }^{41}$ soon after casting. Figure S4 shows that the phase separation speed was reduced for highly concentrated $(6 \%)$ samples. The suspension did not reach the chiral nematic long-range order visible in Figure $5 a_{3}$ during the drying, and consequently, the shearinduced disorder remained in the assembled film. The resulting films had a higher average pitch size and larger variation in pitch (Figure $4 b_{3}$ ). Many of the samples that have been visualized in the literature have been dried from CNC suspensions from initial concentrations below or above the critical concentration for formation of the anisotropic phase. $^{23,24,27,39,41,42,59}$ Films dried from initial concentrations above the critical concentration were presumably assembled from a nonequilibrated suspension, according to route Figure $5 b_{3} \rightarrow 5 b_{4}$. This effectively limited the formation of ordered helical structures and thereby prevented to fully revealing the potential of structural color into large areas. It is well-known that the CNC size and aspect ratio determine the critical concentration for phase separation as well as the gelation concentration. ${ }^{5}$ Thus, these are important considerations in the formation of domains in films formed by EISA, as faster gelation kinetics can lead to larger pitch in the dried films. ${ }^{41}$

3.5. Films Assembled from Fractionated Suspensions. We evaluated the anisotropic and isotropic fractions, which were fractionated from a CNC suspension, to further understand the formation of structured $\mathrm{CN}$ domains in films obtained from high AVF suspensions. The two phases of the $6 \%$ CNC suspension (Figure 6a) were separated after equilibration for 28 days. The isotropic phase was separated after an intermediate liquid volume had been removed to avoid any anisotropic fraction contamination in the separated isotropic (note: we cannot rule out the possibility of some mixing due to the Couette flow, resulting in a portion of the AVF's nanocrystals transferring into the separated isotropic volume fraction). The solids content for each fraction corresponded to $6.1 \%$ (anisotropic) and 5.8\% (isotropic), suggesting a denser anisotropic phase, as has been reported in a previous study. ${ }^{5}$

The CNC size distribution determined for the two phases (Figure $6 b_{2}, c_{2}$ ) clearly indicate smaller average CNC length in the isotropic phase compared to that from the anisotropic phase (Figure $6 b_{1}, c_{1}$ ), as expected. ${ }^{5}$ Furthermore, the polydispersity of the particle size in the lower phase was 

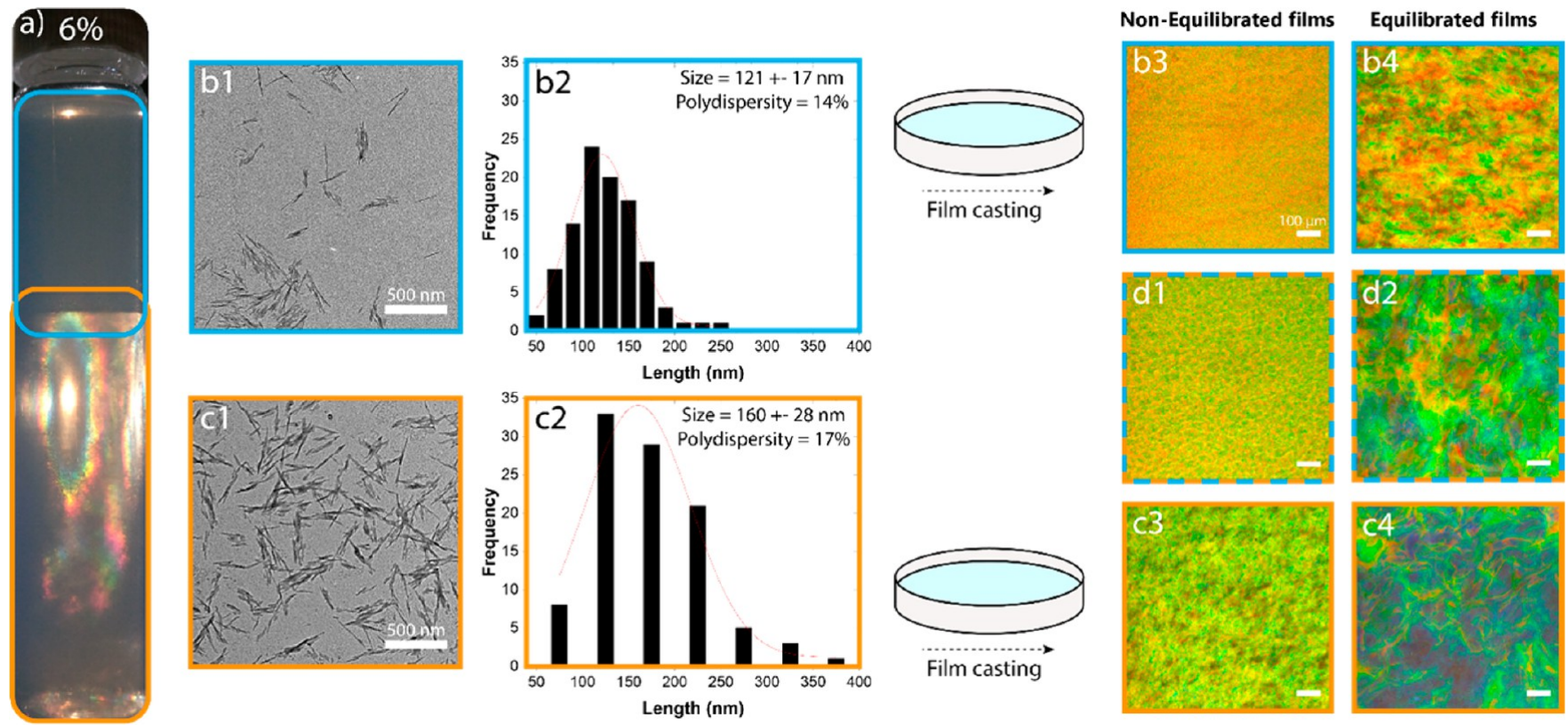

Figure 6. (a) CNC suspension (6\% concentration) in a test tube displaying the top, isotropic and bottom, anisotropic phases. The respective TEM images $\left(b_{1}\right.$ and $\left.c_{1}\right)$ of the nanocrystals in each of these phases are also shown. The CNC length distribution in the $\left(b_{2}\right)$ isotropic and $\left(c_{2}\right)$ anisotropic phase are also included. Optical in-plane reflected-light microscope images of films assembled from the entire suspension are shown in $\left(d_{1}, d_{2}\right)$, corresponding to N6 and E6. $\left(b_{3}, b_{4}\right)$ Correspond to the films formed from the upper, isotropic phase of the suspension after equilibration for 0 ( $b_{3}$, $\left.\mathrm{N}_{\text {iso }} 6\right)$ and 7 days $\left(\mathrm{b}_{4}, E_{\text {iso }} 6\right)$. $\left(c_{3}, c_{4}\right)$ Correspond to the films formed from the bottom, anisotropic phase of the suspension after equilibration for 0 $\left(c_{3}, \mathrm{~N}_{a n} 6\right.$ film) and 7 days $\left(c_{4}, E_{a n} 6\right.$ film).

higher. Similar size differences have been previously observed for fractionated CNC suspensions derived from filter paper ${ }^{5}$ and bacterial cellulose. ${ }^{60}$ Our size analyses revealed CNC widths of $9 \pm 2 \mathrm{~nm}$ in both phases, suggesting that the aspect ratio of the nanocrystals in the bottom phase was higher. Our results are in line with Onsager's ${ }^{61}$ prediction that nanorods of higher aspect ratio form liquid crystals at a lower concentration than those of smaller sizes, thereby offering a possibility for fractionating the CNCs based on size.

The isotropic and anisotropic phases fractionated from the 6\% CNC suspension were each cast into the respective supported films. Those obtained from the isotropic nonequilibrated suspension correspond to $\mathrm{N}_{\text {iso }} 6$ (Figure $6 \mathrm{~b}_{3}$ ), and those from the equilibrated suspensions correspond to $E_{\text {iso }} 6$ (Figure $6 \mathrm{~b}_{4}$ ). Similarly for the anisotropic phases, $\mathrm{N}_{a n} 6$ (Figure $6 c_{3}$ ) and $E_{a n} 6$ (Figure $6 c_{3}$ ). For comparison, films that were obtained from the entire volume of the suspensions without and with equilibration (7 days) are shown in Figure $6 d_{1}(N 6)$ and Figure $6 \mathrm{~d}_{2}$ (E6), respectively.

Revisiting the results from experiments with the 6\% CNC suspension, it was noticeable that the E6 film displayed a segmented structure (as in Figure $4 c_{2}$ ), and in view of the size distribution results (Figure $6 c_{1}, c_{2}$ ), the CNCs of larger size were likely located in the bottom layer of the film while the smaller sizes were in the top layer. The lack of equilibration created a continuous structure in the N6 films (Figure $4 \mathrm{~b}_{2}$ ) where one can assume that different $\mathrm{CNC}$ sizes were mixed and interdigitated. Upon drying, the CNCs with lower size also contributed to the formation of the long-range order in the film. As a result, there was a larger scatter of pitch values (Figure $4 b_{3}$ ) given the contribution of the different CNC sizes. ${ }^{6}$ This is in contrast with E6, where the smaller CNCs formed liquid crystals later during the EISA process, potentially close to the kinetic arrest (gelation) concentration. ${ }^{16,41}$ Consequently, the smaller CNCs formed the disordered upper layer in the film, while the larger size fraction (in the AVF) was assembled in the bottom layer, thus explaining narrower pitch dispersion of the E6 sample (Figure $4 c_{3}$ ).

During the assembly of an equilibrated $6 \%$ suspension, it is possible that the CNCs close to the interface also fractionated in-plane into different domains, in addition to the fractionation in the out-of-plane direction. In this case, any difference in pitch value would arise from $\mathrm{CNC}$ size differences, effectively contributing to the reflection color difference between the domains (Figure $6 \mathrm{~d}_{2}$ ), ${ }^{23}$ in addition to other variables, such as a tilt in the chiral nematic axes. ${ }^{40}$

When examining the microscope images of nonequilibrated films (Figure $6 \mathrm{~b}_{3}, \mathrm{~d}_{1}, \mathrm{c}_{3}$ ), the N6 film (Figure $6 \mathrm{~d}_{1}$ ) can be visualized as resulting from a mixture of the films formed from the separated small (Figure $6 b_{2}$ ) and large (Figure $6 c_{2}$ ) CNCs. Furthermore, in the images of the equilibrated films (Figure $\left.6 \mathrm{~b}_{4}, \mathrm{~d}_{2}, \mathrm{c}_{4}\right)$, it is apparent that the color arises from both the smaller fraction CNCs (Figure $6 b_{2}$ ) in the top part of the film and the larger fraction CNCs of the bottom layer (Figure $6 c_{2}$ ).

As discussed before, equilibration of CNC suspensions above the critical concentration promoted the formation of long-range order in dried films. To this end, the equilibration was expected to facilitate long-range order in the films assembled from the fractionated AVF obtained from the 6\% CNC suspension, which was clearly visible by the larger $\mathrm{CN}$ domains of Figure $6 c_{4}$ compared to those in Figure $6 c_{3}$. Interestingly, films dried from the fully isotropic CNC suspensions, below the critical concentration, did exhibit a variation in $\mathrm{CN}$ domains due to equilibration. Incomplete fractionation and the presence of a small amount of anisotropic volume fraction in the suspension prior to drying could explain the presence of the larger domains observed in Figure $6 d_{4}$ compared to those in Figure $6 d_{3}$. 
Kinetic arrest ${ }^{16,41}$ is expected to occur earlier in a suspension with larger $\mathrm{CNCs},{ }^{5}$ which gel at lower concentrations compared to smaller ones. ${ }^{16}$ Hence, the isotropic phase containing the smaller CNCs would experience kinetic arrest later than the mixture of the phases, giving it more time to assemble into a higher order structure that should exhibit proportionally lower pitch sizes. However, the CNCs in a liquid crystalline system are aligned to some degree, which could effectively limit the effect of rod dimensions on the gelling behavior. Although, to the best of our knowledge, so far, no systematic evaluation has been carried out regarding the effect of long-range order in CNCs suspensions on gelation kinetics. Therefore, the film formed from the isotropic phase (Figure $6 b_{3}$ ), comprising the smaller CNCs, did not exhibit a blueshifted reflection compared to the film assembled from the mixture of the phases (Figure $6 \mathrm{~d}_{1}$ ). This indicates that rather than using smaller CNCs, the extent of equilibration of the liquid crystals suspensions is a dominant variable in creating higher ordered materials with lower pitch size. Additionally, it may be important to consider the difference in morphological anisotropy of CNCs in the isotropic and anisotropic phases. The difference in average length (120 and $160 \mathrm{~nm}$ ) and the similar width $(9 \mathrm{~nm})$ results in a $33 \%$ decrease in morphological anisotropy for CNCs in the isotropic phase.

\section{CONCLUSIONS}

The helical order in dry films formed from aqueous suspensions of cellulose nanocrystals (CNCs) was determined as a function of the anisotropic volume fraction (AVF) of the suspensions. The AVF increased as a function of concentration and comprised CNCs of a larger aspect ratio, which phase-separated at a lower concentration compared to that required for smaller CNCs. Furthermore, the AVF decreased as a function of equilibration time.

The combined effect of high initial AVF and equilibration prior to EISA favored the formation of long-range ordered chiral nematic structures and large merged domains (UV-vis spectroscopy, optical microscopy, and SEM). The larger CNCs present in the AVF of the suspension formed a highly structured, low pitch, bottom layer, whereas the smaller $\mathrm{CNCs}$ assembled into a disordered, top-most layer. Large chiral nematic domains sandwiched between the bottomordered and top-disordered segments likely formed from merging tactoids in the neighborhood of the isotropic/ anisotropic interface during EISA. In contrast, nonequilibrated suspensions resulted in films lacking full color-generating capacity (and displayed wider spectra in UV-vis reflection). These findings stress the importance of a sufficient time window between phase separation at the critical concentration and gelation stages in order to develop tactoids, and subsequently, long-range helical order. The importance of equilibration at high AVF is further highlighted by the fact that equilibration had no significant effect on fully isotropic suspensions prior to drying into films. It is important to note that the inherent physical-chemical characteristics of the CNCs (depending on source, isolation and processing conditions) affect the concentration-dependent AVF and, potentially, the transition of the long-range order from suspensions to dry films. Nevertheless, the formation of chiral nematic order and domains addressed in this work was examined principally in the context of the AVF, thus extending its usefulness into other cellulose-based liquid crystalline systems, e.g., bacterial cellulose nanocrystals. Further compar- ison would be of high interest in order to develop, for instance, charge- or size-dependent scaling rules for the transitions from long-range ordered suspensions to hierarchically structured materials. Our results further the understanding of the formation of helical order, essential for creating next-generation materials, such as impact resistant structural elements. This also applies to the formation of materials with long-range order, exploiting anisotropy or domains across the film in-plane and out-of-plane dimensions. Finally, the role of both the critical concentration and the kinetic arrest concentration required for the development of liquid crystals in films formed by EISA should be underscored.

\section{ASSOCIATED CONTENT}

\section{Supporting Information}

The Supporting Information is available free of charge on the ACS Publications website at DOI: 10.1021/acs.biomac.8b00497.

Physicochemical properties of CNCs (zeta potential, hydrodynamic radius, $\mathrm{pH}$ of the suspension); anisotropic volume fractions of cellulose nanocrystal suspensions as a function of concentration $(w / w)$ and equilibration time; UV-vis transmission spectra of the N3, N4, N5, N6, E3, E4, E5, and E6 films; microscope images and UV-vis transmission spectra of a parallel set of films to the above-mentioned, but with alternative substrate and equilibration time; supporting scanning electron microscopy images of the E6 sample (PDF)

\section{AUTHOR INFORMATION}

\section{Corresponding Authors}

*(B.L.T.) E-mail blaise.tardy@aalto.fi.

*(O.J.R.) E-mail orlando.rojas@aalto.fi.

ORCID $\odot$

Blaise L. Tardy: 0000-0002-7648-0376

Anurodh Tripathi: 0000-0002-3415-0221

Orlando J. Rojas: 0000-0003-4036-4020

\section{Notes}

The authors declare no competing financial interest.

\section{ACKNOWLEDGMENTS}

We acknowledge funding support by the Academy of Finland under the Project SIRAF and the European Research Commission for O.J.R. ERC Advanced Grant. The Aalto University Materials Platform is also thanked for funding the MSc thesis of K.W.K. under the project LiCOS. We also appreciate the discussions with colleagues in the School of ARTS, Noora Yau and Jaana Beidler. The authors are thankful to the Academy of Finland through its Centres of Excellence Programme (2014-2019) under Project 264677 "Molecular Engineering of Biosynthetic Hybrid Materials Research" (HYBER).

\section{ABBREVIATIONS}

$\mathrm{AVF}$, anisotropic volume fraction; $\mathrm{CN}$, chiral nematic; $\mathrm{CNC}$, cellulose nanocrystal; SEM, scanning electron microscopy; TEM, transmission electron microscopy.

\section{REFERENCES}

(1) Fox, J. D.; Capadona, J. R.; Marasco, P. D.; Rowan, S. J. Bioinspired Water-Enhanced Mechanical Gradient Nanocomposite 
Films That Mimic the Architecture and Properties of the Squid Beak. J. Am. Chem. Soc. 2013, 135, 5167-5174.

(2) Weaver, J. C.; Milliron, G. W.; Miserez, A.; Evans-Lutterodt, K.; Herrera, S.; Gallana, I.; Mershon, W. J.; Swanson, B.; Zavattieri, P.; DiMasi, E.; Kisailus, D. The Stomatopod Dactyl Club: A Formidable Damage-Tolerant Biological Hammer. Science 2012, 336, 1275-1280.

(3) Habibi, Y.; Lucia, L. A.; Rojas, O. J. Cellulose Nanocrystals: Chemistry, Self-Assembly, and Applications. Chem. Rev. 2010, 110, 3479-3500.

(4) Roman, M. Toxicity of Cellulose Nanocrystals: A Review. Ind. Biotechnol. 2015, 11, 25-33.

(5) Min Dong, X.; Revol, J.; Gray, G. D. Effect of microcrystallite preparation conditions on the formation of colloid crystals of cellulose. Cellulose 1998, 5, 19-32.

(6) Beck-Candanedo, S.; Roman, M.; Gray, G. D. Effect of Reaction Conditions on the Properties and Behavior of Wood Cellulose Nanocrystal Suspensions. Biomacromolecules 2005, 6, 1048-1054.

(7) Agarwal, U.; et al. Tailoring the yield and characteristics of wood cellulose nanocrystals (CNC) using concentrated acid hydrolysis. Cellulose 2015, 22, 1753-1762.

(8) Favier, V.; Chanzy, H.; Cavaille, J. Y. Polymer Nanocomposites Reinforced by Cellulose Whiskers. Macromolecules 1995, 28, 63656367.

(9) Šturcová, A.; Davies, G. R.; Eichhorn, S. J. Elastic Modulus and Stress-Transfer Properties of Tunicate Cellulose Whiskers. Biomacromolecules 2005, 6, 1055-1061.

(10) Siqueira, G.; Bras, J.; Dufresne, A. Cellulose Whiskers versus Microfibrils: Influence of the Nature of the Nanoparticle and its Surface Functionalization on the Thermal and Mechanical Properties of Nanocomposites. Biomacromolecules 2009, 10, 425-432.

(11) Saito, T.; Kuramae, R.; Wohlert, J.; Berglund, L. A.; Isogai, A. An Ultrastrong Nanofibrillar Biomaterial: The Strength of Single Cellulose Nanofibrils Revealed via Sonication-Induced Fragmentation. Biomacromolecules 2013, 14, 248-253.

(12) Iwamoto, S.; Kai, W.; Isogai, A.; Iwata, T. Elastic Modulus of Single Cellulose Microfibrils from Tunicate Measured by Atomic Force Microscopy. Biomacromolecules 2009, 10, 2571-2576.

(13) Gray, G. D.; Mu, X. Chiral Nematic Structure of Cellulose Nanocrystal Suspensions and Films; Polarized Light and Atomic Force Microscopy. Materials 2015, 8, 7873-7888.

(14) Nguyen, T.; MacLachlan, M. J. Biomimetic Chiral Nematic Mesoporous Materials from Crab Cuticles. Adv. Opt. Mater. 2014, 2, 1031-1037.

(15) Honorato-Rios, C.; Kuhnhold, A.; Bruckner, J. R.; Dannert, R.; Schilling, T.; Lagerwall, J. P. F. Equilibrium Liquid Crystal Phase Diagrams and Detection of Kinetic Arrest in Cellulose Nanocrystal Suspensions. Front. Mater. 2016, 3, 1-13.

(16) Lagerwall, J. P. F.; Schütz, C.; Salajkova, M.; Park, J. H.; Scalia, G.; Bergstrom, L. Cellulose nanocrystal-based materials: from liquid crystal self-assembly and glass formation to multifunctional thin films. NPG Asia Mater. 2014, 6, 1-12.

(17) Isogai, A.; Saito, T.; Fukuzumi, H. TEMPO-oxidized cellulose nanofibers. Nanoscale 2011, 3, 71-85.

(18) Wang, B.; Walther, A. Self-Assembled, Iridescent, CrustaceanMimetic Nanocomposites with Tailored Periodicity and Layered Cuticular Structure. ACS Nano 2015, 9, 10637-10646.

(19) Tatsumi, M.; Teramoto, Y.; Nishio, Y. Different orientation patterns of cellulose nanocrystal films prepared from aqueous suspensions by shearing under evaporation. Cellulose 2015, 22, 2983-2992.

(20) Liu, D.; Li, J.; Sun, F.; Xiao, R.; Guo, Y.; Song, J. Liquid crystal microphase separation of cellulose nanocrystals in wet-spun PVA composite fibers. RSC Adv. 2014, 4, 30784-30789.

(21) Guidetti, G.; Atifi, S.; Vignolini, S.; Hamad, W. Y. Flexible Photonic Cellulose Nanocrystal Films. Adv. Mater. 2016, 28, 1004210047.

(22) Wang, P.; Hamad, W. Y.; MacLachlan, M. J. Structure and transformation of tactoids in cellulose nanocrystal suspensions. Nat. Commun. 2016, 7, 11515.
(23) Dumanli, A. G.; van der Kooij, H. M.; Kamita, G.; Reisner, E.; Baumberg, J. J.; Steiner, U.; Vignolini, S. Digital Color in Cellulose Nanocrystal Films. ACS Appl. Mater. Interfaces 2014, 6, 12302-12306.

(24) Park, J. H.; Noh, J.; Schutz, C.; Salazar-Alvarez, G.; Scalia, G.; Bergstrom, L.; Lagerwall, J. P. F. Macroscopic Control of Helix Orientation in Films Dried from Cholesteric Liquid-Crystalline Cellulose Nanocrystal Suspensions. ChemPhysChem 2014, 15, 14771484.

(25) Tran, A.; Hamad, W. Y.; MacLachlan, M. J. Tactoid Annealing Improves Order in Self-Assembled Cellulose Nanocrystal Films with Chiral Nematic Structures. Langmuir 2018, 34, 646-652.

(26) Dong, X. M.; Kimura, T.; Revol, J.; Gray, D. G. Effects of Ionic Strength on the Isotropic-Chiral Nematic Phase Transition of Suspensions of Cellulose Crystallites. Langmuir 1996, 12, 2076-2082.

(27) Ličen, M.; Majaron, B.; Noh, J.; Schütz, C.; Bergström, L.; Lagerwall, J.; Drevenšek-Olenik, I. Correlation between structural properties and iridescent colors of cellulose nanocrystalline films. Cellulose 2016, 23, 3601-3609.

(28) Tardy, B. L.; Ago, M.; Guo, J.; Borghei, M.; Kämäräinen, T.; Rojas, O. J. Optical properties of self-assembled cellulose nanocrystals films suspended at planar-symmetrical interfaces. Small 2017, 13, 1702084 .

(29) Nguyen, T.; Hamad, W. Y.; MacLachlan, M. J. Tuning the iridescence of chiral nematic cellulose nanocrystals and mesoporous silica films by substrate variation. Chem. Commun. 2013, 49, 1129611298.

(30) Dumée, L.; Lee, J.; Sears, K.; Tardy, B. L.; Duke, M.; Gray, S. Fabrication of thin film composite poly(amide)-carbon-nanotubes supported membranes for enhanced performance in osmotically driven desalination systems. J. Membr. Sci. 2013, 427, 422-430.

(31) Reid, M. S.; Villalobos, M.; Cranston, E. D. Benchmarking Cellulose Nanocrystals: From the Laboratory to Industrial Production. Langmuir 2017, 33, 1583-1598.

(32) Zoppe, J. O.; Dupire, A. V. M.; Lachat, T. G. G.; Lemal, P.; Rodriguez-Lorenzo, L.; Petri-Fink, A.; Weder, C.; Klok, H. Cellulose Nanocrystals with Tethered Polymer Chains: Chemically Patchy versus Uniform Decoration. ACS Macro Lett. 2017, 6, 892-897.

(33) Smyth, M.; M’Bengue, M.; Terrien, M.; Picart, C.; Bras, J.; Foster, J. E. The effect of hydration on the material and mechanical properties of cellulose nanocrystal-alginate composites. Carbohydr. Polym. 2018, 179, 186-195.

(34) Du, L.; Zhong, T.; Wolcott, M. P.; Zhang, Y.; Qi, C.; Zhao, B.; Wang, J.; Yu, Z. Dispersing and stabilizing cellulose nanoparticles in acrylic resin dispersions with unreduced transparency and changed rheological property. Cellulose 2018, 25, 2435-2450.

(35) Tardy, B. L.; Tan, S.; Dam, H. H.; Ejima, H.; Blencowe, A.; Qiao, G. G.; Caruso, F. Nanoparticles assembled via $\mathrm{pH}$-responsive reversible segregation of cyclodextrins in polyrotaxanes. Nanoscale 2016, 8, 15589-15596.

(36) Majoinen, J.; Kontturi, E.; Ikkala, O.; Gray, D. G. SEM imaging of chiral nematic films cast from cellulose nanocrystal suspensions. Cellulose 2012, 19, 1599-1605.

(37) Ureña-Benavides, E. E.; Ao, G.; Davis, V. A.; Kitchens, C. L. Rheology and Phase Behavior of Lyotropic Cellulose Nanocrystal Suspensions. Macromolecules 2011, 44, 8990-8998.

(38) Abitbol, T.; Kam, D.; Levi-Kalisman, Y.; Gray, D. G.; Shoseyov, $\mathrm{O}$. Surface Charge Influence on the Phase Separation and Viscosity of Cellulose Nanocrystals. Langmuir 2018, 34, 3925-3933.

(39) Gençer, A.; Schütz, C.; Thielemans, W. Influence of the Particle Concentration and Marangoni Flow on the Formation of Cellulose Nanocrystal Films. Langmuir 2017, 33, 228-234.

(40) de Vries, H. Rotatory Power and other Optical Properties of Certain Liquid Crystals. Acta Crystallogr. 1951, 4, 219-226.

(41) Mu, X.; Gray, D. G. Formation of Chiral Nematic Films from Cellulose Nanocrystal Suspensions Is a Two-Stage Process. Langmuir 2014, 30, 9256-9260.

(42) Mu, X.; Gray, D. G. Droplets of cellulose nanocrystal suspensions on drying give iridescent 3-D "coffee-stain" rings. Cellulose 2015, 22, 1103-1107. 
(43) Beck-Candanedo, S.; Bouchard, J.; Chauve, G.; Berry, R. Controlled production of patterns in iridescent solid films of cellulose nanocrystals. Cellulose 2013, 20, 1401-1411.

(44) Liu, D.; Wang, S.; Ma, Z.; Tian, D.; Gu, M.; Lin, F. Structurecolor mechanism of iridescent cellulose nanocrystal films. RSC Adv. 2014, 4, 39322-39331.

(45) Pan, J.; Hamad, W. Y.; Straus, S. K. Parameters Affecting the Chiral Nematic Phase of Nanocrystalline Cellulose Films. Macromolecules 2010, 43, 3851-3858.

(46) Beck, S.; Bouchard, J.; Berry, R. Controlling the Reflection Wavelength of Iridescent Solid Films of Nanocrystalline Cellulose. Biomacromolecules 2011, 12, 167-172.

(47) Tang, H.; Guo, B.; Jiang, H.; Xue, L.; Li, B.; Cao, X.; Zhang, Q.; $\mathrm{Li}, \mathrm{P}$. Fabrication and characterization of nanocrystalline cellulose films prepared under vacuum conditions. Cellulose 2013, 20, 2667-2674.

(48) Bodiguel, H.; Leng, J. Imaging the drying of a colloidal suspension. Soft Matter 2010, 6, 5451-5460.

(49) Gray, D. G.; Mu, X. Twist-Bend Stage in the Relaxation of Sheared Chiral Nematic Suspensions of Cellulose Nanocrystals. ACS Omega 2016, 1, 212-219.

(50) Shafiei-Sabet, S.; Hamad, W. Y.; Hatzikiriakos, S. G. Rheology of Nanocrystalline Cellulose Aqueous Suspensions. Langmuir 2012, 28, 17124-17133.

(51) Dumanli, A. G.; Kamita, G.; Landman, J.; van der Kooij, H.; Glover, B. J.; Baumberg, J. J.; Steiner, U.; Vignolini, S. Controlled, Bioinspired Self-Assembly of Cellulose-Based Chiral Reflectors. Adv. Opt. Mater. 2014, 2, 646-650.

(52) Klemm, D.; Philipp, B.; Heinze, T.; Heinze, U.; Wagenknecht, W. General Considerations on Structure and Reactivity of Cellulose: Section 2.1-2.1.4 In Comprehensive Cellulose Chemistry; Wiley-VCH Verlag GmbH \& Co. KGaA: 2004; pp 9-29.

(53) Chen, Q.; Liu, P.; Nan, F.; Zhou, L.; Zhang, J. Tuning the Iridescence of Chiral Nematic Cellulose Nanocrystal Films with a Vacuum-Assisted Self-Assembly Technique. Biomacromolecules 2014, $15,4343-4350$.

(54) Bardet, R.; Roussel, F.; Coindeau, S.; Belgacem, N.; Bras, J. Engineered pigments based on iridescent cellulose nanocrystal films. Carbohydr. Polym. 2015, 122, 367-375.

(55) Bardet, R.; Belgacem, N.; Bras, J. Flexibility and Color Monitoring of Cellulose Nanocrystal Iridescent Solid Films Using Anionic or Neutral Polymers. ACS Appl. Mater. Interfaces 2015, 7, $4010-4018$.

(56) Jativa, F.; Schutz, C.; Bergstrom, L.; Zhang, X.; Wicklein, B. Confined self-assembly of cellulose nanocrystals in a shrinking droplet. Soft Matter 2015, 11, 5374-5380.

(57) Revol, J.; Bradford, H.; Giasson, J.; Marchessault, R. H.; Gray, D. G. Helicoidal self-ordering of cellulose microfibrils in aqueous suspension. Int. J. Biol. Macromol. 1992, 14, 170-172.

(58) Chen, W.; Gray, D. G. Interfacial Tension between Isotropic and Anisotropic Phases of a Suspension of Rodlike Particles. Langmuir 2002, 18, 633-637.

(59) Zhang, Y. P.; Chodavarapu, V. P.; Kirk, A. G.; Andrews, M. P. Structured color humidity indicator from reversible pitch tuning in self-assembled nanocrystalline cellulose films. Sens. Actuators, B 2013, $176,692-697$.

(60) Hirai, A.; Inui, O.; Horii, F.; Tsuji, M. Phase Separation Behavior in Aqueous Suspensions of Bacterial Cellulose Nanocrystals Prepared by Sulfuric Acid Treatment. Langmuir 2009, 25, 497-502.

(61) Onsager, L. The effects of shape on the interactions of colloidal particles. Ann. N. Y. Acad. Sci. 1949, 51, 627-659. 